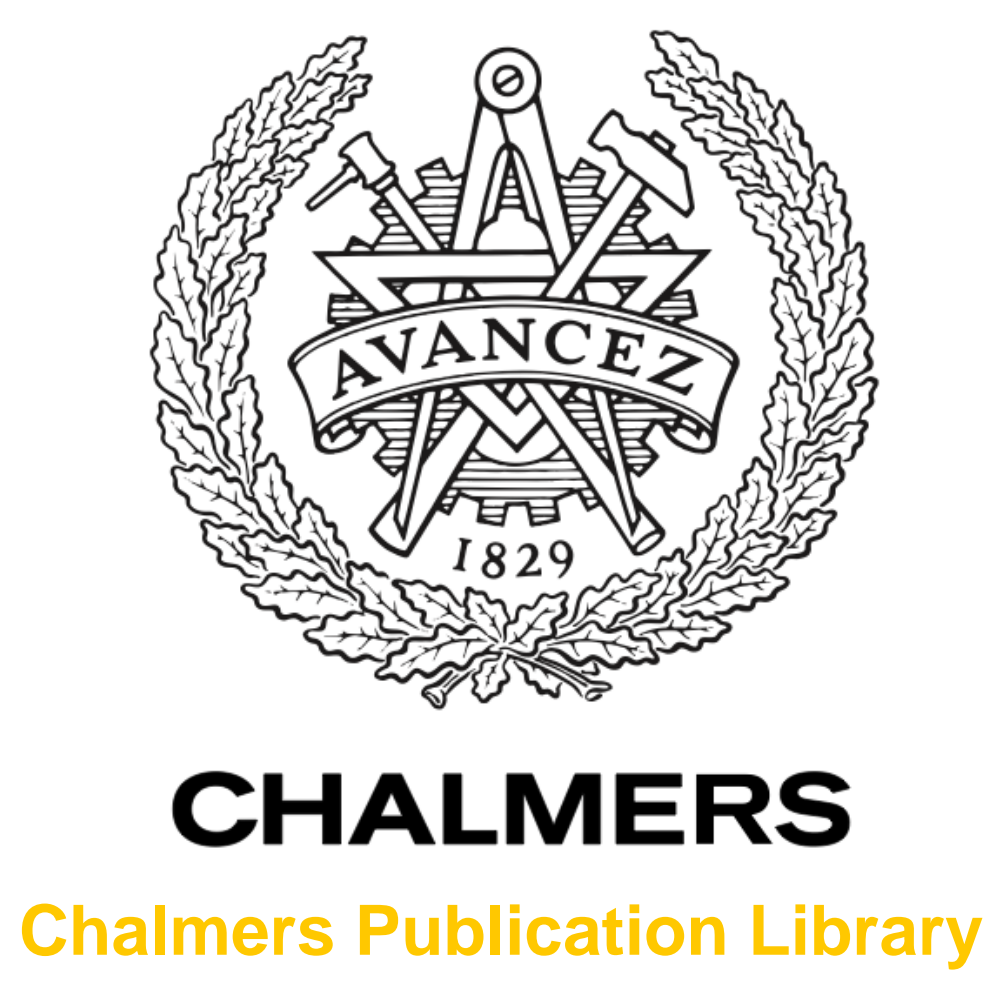

Modeling and reconstruction in a 3D microwave imaging system

This document has been downloaded from Chalmers Publication Library (CPL). It is the author's version of a work that was accepted for publication in:

30th URSI General Assembly and Scientific Symposium, URSIGASS 2011, Istanbul, 13-20 August 2011

Citation for the published paper:

Fhager, A. ; Köster, J. ; Rubaek, T. (2011) "Modeling and reconstruction in a 3D microwave imaging system". 30th URSI General Assembly and Scientific Symposium, URSIGASS

2011, Istanbul, 13-20 August 2011

http://dx.doi.org/10.1109/URSIGASS.2011.605044

8

Down「oaded from: http://publications.lib.chalmers.se/publication/150865

Notice: Changes introduced as a result of publishing processes such as copy-editing and formatting may not be reflected in this document. For a definitive version of this work, please refer to the published source. Please note that access to the published version might require a subscription. 


\title{
Modeling and Reconstruction in a 3D Microwave Imaging System
}

\author{
Andreas Fhager, Johan Köster, Tonny Rubak, and Mikael Persson \\ Department of Signals and Systems, Chalmers University of Technology, SE-412 96 Gothenburg, Sweden, \\ andreas.fhager@chalmers.se, rubaek@chalmers.se, mikael.persson@chalmers.se
}

\begin{abstract}
In this paper we discuss the design and modeling of a 3D system for microwave imaging. The antenna system consists of 32 monopoles in a cylindrical configuration. A FDTD model of the system is used in the imaging reconstruction algorithm. Here we show a comparison of the data generated from the numerical FDTD model with measured data, as well as imaging of a simple target using this system. The agreement in the model is good and the imaging of a test object is successful.
\end{abstract}

\section{Introduction}

Breast cancer is a serious health problem for women. According to national statistics of Sweden from year 2007 it is the most common cause of death from cancer among women, with about 30 yearly deaths per 100,000 inhabitants, [1]. The situation is similar in the rest of Europe, Northern America and Australia, [2].

Today the most common method for breast cancer screening and diagnosis is X-ray mammography. It is well recognized for its ability to detect tumors but it also suffers from some disturbing limitations. Neither the false positive nor the false negative detection rates are negligible. This leads to a number of unnecessary additional investigations and, more seriously, a fraction of the tumors are not detected at an early stage which is a prerequisite for efficient treatment. A major reason for the limitations using a technique based on X-rays is that the contrast between the tumor and the surrounding tissue sometimes can be as low as a few percent.

An interesting alternative that could potentially remedy some of the drawbacks with X-ray is imaging with a technique based on microwaves. In dielectric measurements a significant contrast has been observed between healthy and malignant tissue at these frequencies. Depending on the mixture between fatty and glandular tissue in the breast the contrast varies largely between individual patients, from about $10 \%$ for a breast with a large portion of glandular tissue and up to $>100 \%$ for breasts consisting of mostly fatty tissue, [3]. In recent clinical studies the ability to detect breast cancer tumors with microwaves have been shown, [4]. In this paper we present results from our work with developing a 3D clinical imaging prototype.

\section{Methods for Image Reconstruction and Measurements}

In microwave tomography the object under investigation is surrounded by a number of transmitting and receiving antennas. In the measurements each antenna is operated as a transmitter as well as a receiver for every possible combination of antennas. The basic idea for the image reconstruction algorithm is to compare the measured data with a corresponding numerical simulation of the system. Based on the difference between the measured and the simulated signals the object under reconstruction is iteratively refined in the computational model. As the reconstruction of the target object is iteratively refined, the simulated and measured signals converge. The assumption is that when the difference is sufficiently small the reconstruction is completed. In our work we utilize wide band pulses as for the imaging and we use a time domain formulation of the reconstruction procedure in which the aim is to minimize the objective functional, $F$, defined as

$$
F(\epsilon, \sigma)=\int_{0}^{T} \sum_{m=1}^{M} \sum_{n=1}^{N}\left|\mathbf{E}_{m}\left(\epsilon, \sigma, \mathbf{R}_{n}, t\right)-\mathbf{E}_{m}^{m}\left(\mathbf{R}_{n}, t\right)\right|^{2} d t,
$$


where $\mathbf{E}_{m}\left(\epsilon, \sigma, \mathbf{R}_{n}, t\right)$ is the calculated field from the computational model and $\mathbf{E}_{m}^{m}\left(\mathbf{R}_{n}, t\right)$ is the corresponding measured data when antenna number $m$ has been used as transmitter. Here $M$ is the number of transmitters, $N$ is the number of receivers and $\mathbf{R}_{n}$ denotes the position of antenna number $n$.

In much of the published work as well as in most of our own work imaging has been restricted to 2D antenna arrays and $2 \mathrm{D}$ reconstruction algorithms. The main motivation for using $2 \mathrm{D}$ is to reduce the computational burden, which easily can grow out of control in a 3D configuration. Consequently we have earlier used a flat antenna array, where the antennas have been placed on a circle together with a 2D reconstruction algorithm, [5]- [8]. However, this setup is also most suitable for imaging 2D objects with dielectric properties that are constant in the direction perpendicular to the antenna plane. When it comes to imaging $3 \mathrm{D}$ objects the accuracy is seriously limited. The reason for this is at least twofold. Number one; it is not possible to make accurate models of the real world antennas in a $2 \mathrm{D}$ algorithm. Number two; the propagation model of the real world 3D wave pattern in a $2 \mathrm{D}$ model is not accurate. This introduces serious limitations in the accuracy of the simulated data and which will manifest itself in the comparison with the measured data. We have also developed a 3D algorithm that we have used with this measurement setup, [9]. In this algorithm we can make accurate models of the antennas but since we have no antennas outside the plane we still can get no information about the objects in the z-direction and therefore still only image $2 \mathrm{D}$ objects.

In our current strive to develop a clinical prototype we have therefore concluded that the most suitable design of a clinical prototype is to use an antenna array where monopole antennas are placed in a 3D pattern. We have chosen to construct a cylindrical antenna array and together with a fully 3D reconstruction software the potential for improved accuracy is increased. The price that has to be paid for this approach is a significantly increased computational load in the reconstruction algorithm. The principal design of the antenna array under investigation can be found in Fig. 1(a) together with a photo in (b). The diameter of the cylindrical antenna array is $140 \mathrm{~mm}$ and the height is $120 \mathrm{~mm}$. The diameter of the plastic tank is 400 mm.

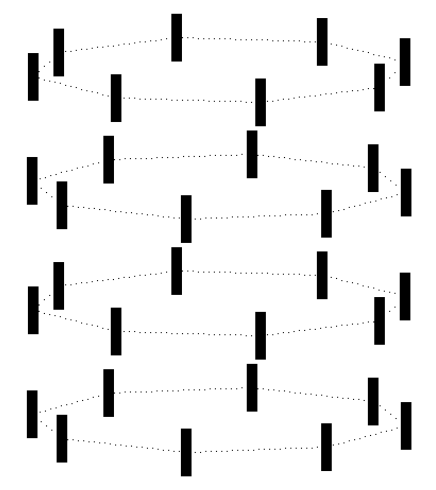

(a) Antenna array configuration

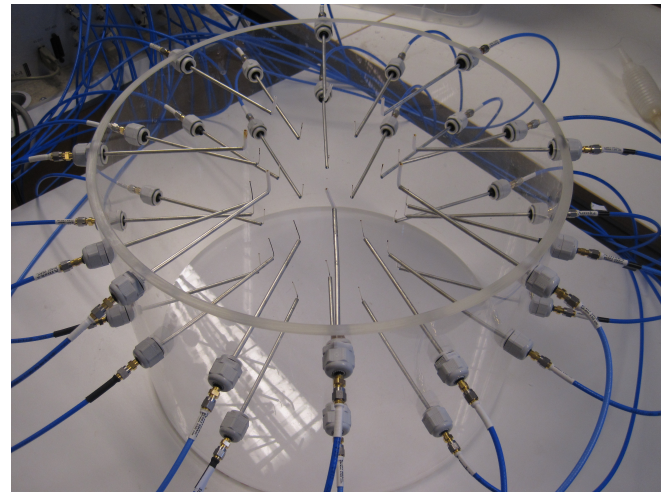

(b) Photo of antenna array

Figure 1: The principal design of the antenna array. The antenna array consists of 32 antennas in total, configured in four circles with eight antennas in each circle. Also shown is a photo of the experimental system.

\section{Results}

In the strive to evaluate the performance of this system several imaging scenarios have been evaluated and will be evaluated. The foundation for all imaging is that an accurate electromagnetic model can be obtained such that an accurate comparison with measured data can be made. In this work we use a FDTD model based on a thin-wire approximation to model the antenna wire and the resistive voltage source to 
model the feed. In Fig. 2 two selected reflection and transmission coefficients have been plotted, both measured data and simulated data has been plotted in order to evaluate the accuracy of the numerical model. The agreement between the two curves is good, and similar agreement can be seen also for the rest of the antennas.

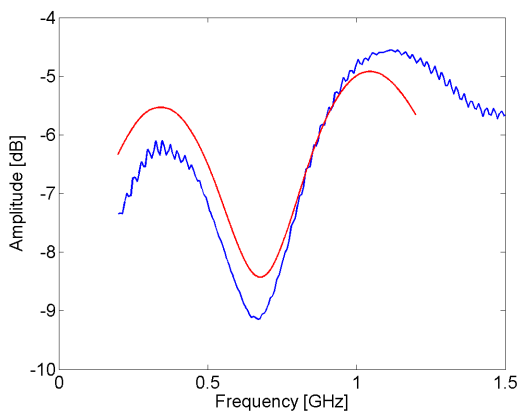

(a) Reflection

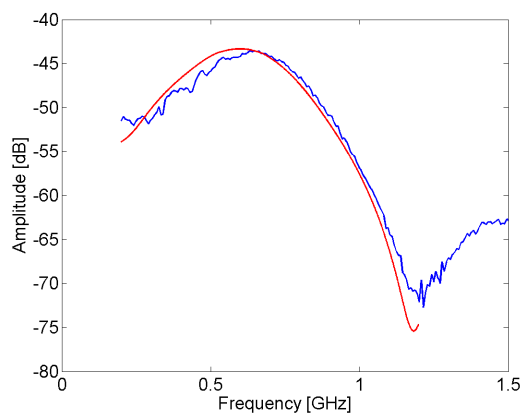

(b) Transmission

Figure 2: This plot shows measured (blue) and simulated (red) S-parameter data for two selected antennas in the array. To the left a reflection coefficient and to the right the transmission coefficient for two neighboring antennas.

Using this FDTD model we have reconstructed a simple target in form of a plastic cylinder. In this case the tank was filled with a saline solution having $\epsilon_{r}=78$ and $\sigma=1.1 \mathrm{~S} / \mathrm{m}$ and the plastic cylinder was immersed into the array from above. Scattering parameters were measured and the image reconstructed. Shown in Fig. 3 are a representation of the original model together with reconstructions of the permittivity and conductivity for both measured and simulated data. We see that the reconstructions of the permittivity

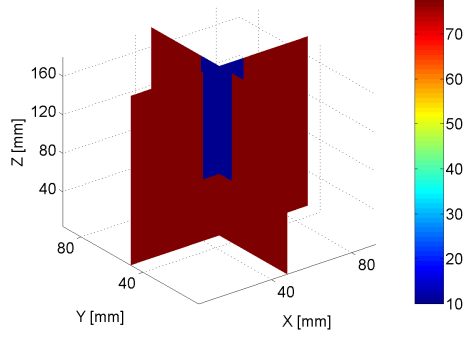

(a) Original relative permittivity

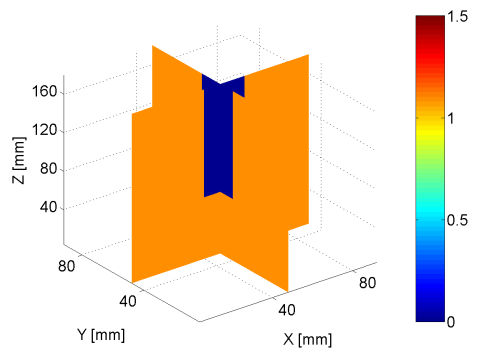

(d) Original conductivity

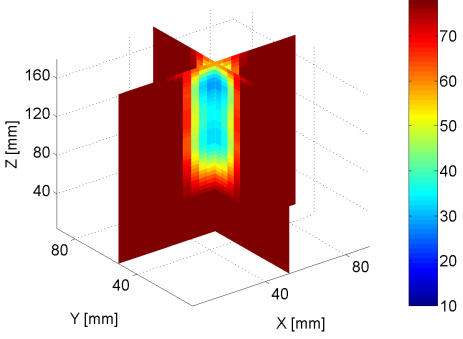

(b) Reconstructed $\epsilon_{r}$ simulated data

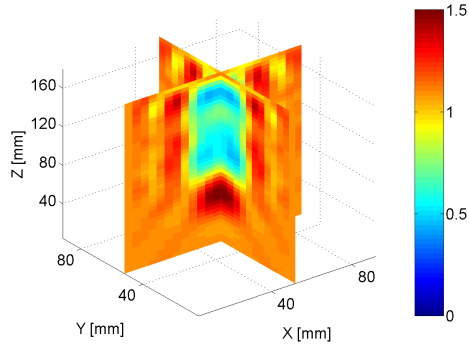

(e) Reconstructed $\sigma$, simulated data

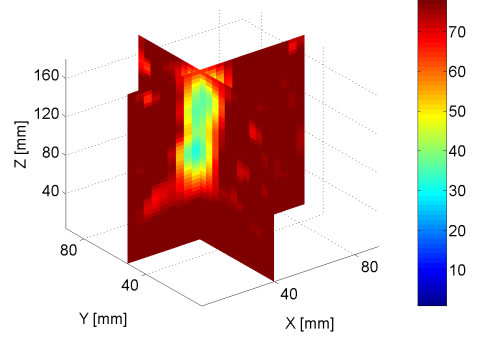

(c) Reconstructed $\epsilon_{r}$, measured data

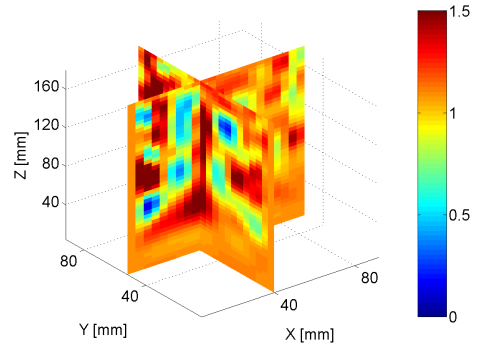

(f) Reconstructed $\sigma$, measured data

Figure 3: Representation of the original model and reconstructions from measured and simulated data.

based on both experimental and measured data agree well with the original model. However the conductivity shows significant artifacts already for the simulated data. Consequently the reconstruction from experimental 
data is even worse, with large artifacts and no clear recovery of the original object.

\section{Conclusion}

In this paper we have described a 3D system for microwave imaging. A FDTD model of the system was made and the results from this numerical model have been shown to agree well with the corresponding measured data. Furthermore reconstruction of a simple target originating from measured and simulated data was shown. The result was found to be more accurate for the permittivity than for the conductivity.

\section{Acknowledgments}

This work was supported in part by VINNOVA within the VINN Excellence Center Chase, and in part by SSF within the Strategic Research Center Charmant. Funding from KG Eliassons tilläggsfond for covering

the costs of the imaging prototype is gratefully acknowledged. The computations were performed on the C3SE computing resources.

\section{References}

[1] Sweden's National Board of Health and Welfare: "Causes of death 2007", (Access at: www.sos.se).

[2] D. M. Parkin, F. Bray, J. Ferlay and P. Pisani: "Global cancer statistics, 2002," CA: A Cancer Journal for Clinicians, vol. 55, pp. 74-108, Mar. 2005.

[3] M. Lazebnik, D. Popovic, L. McCartney, C.B. Watkins, M.J. Lindstrom, J. Harter, S. Sewall, T. Ogilvie, A. Magliocco, T.M. Breslin, W. Temple, D. Mew, J.H. Booske, M. Okoniewski, and S.C. Hagness: "A large-scale study of the ultrawideband microwave dielectric properties of normal, benign, and malignant breast tissues obtained from cancer surgeries," Physics in Medicine and Biology, vol. 52, pp. 6093-6115, October 2007.

[4] S.P. Poplack, T.D. Tosteson, W.A. Wells, B.W Pogue, P.M. Meaney, A. Hartov, C.A. Kogel, S.K. Soho, J.J. Gibson, and K.D. Paulsen: "Electromagnetic Breast Imaging: Results of a Pilot Study in Women with Abnormal Mammograms," Radiology, vol. 243, pp. 350-359, 2007.

[5] P. Hashemzadeh, A. Fhager, and M. Persson, "Experimental investigation of an optimization approach to microwave tomography," Electromagn. Biol. Med., vol. 25, no. 1, pp. 1-12, 2006.

[6] A. Fhager, P. Hashemzadeh and M. Persson, "Reconstruction quality and spectral content of an electromagnetic time-domain inversion algorithm," IEEE Trans. Biomed. Eng., vol. 53, no. 8, pp. 1594-1604, Aug. 2006.

[7] A. Fhager, and M. Persson, "Using a priori Data to Improve the Reconstruction of Small Objects in Microwave Tomography," IEEE Trans. Microwave Theory Tech., vol. 55, pp. 2452-2462, 2007.

[8] S. Nordebo, A. Fhager, M. Gustafsson and M. Persson, "A Systematic Approach to Robust Preconditioning for Gradient-Based Inverse Scattering Algorithms," Inverse Prob., vol. 24, no. 2, art.no. 025027, April, 2008.

[9] S. K. Padhi, A. Fhager, M. Persson and J. Howard, "Measured Antenna Response of a Proposed Microwave Tomography System Using an Efficient 3D-FDTD Model," IEEE Antennas Wirel. Propag. Lett., vol. 7, pp. 689-692, 2008. 